\title{
Determining the nuclear data uncertainty on MONK10 and WIMS10 criticality calculations
}

\author{
Tim Ware ${ }^{\mathrm{a}}$, Geoff Dobson, David Hanlon, Richard Hiles, Robert Mason, and Ray Perry \\ ANSWERS Software Service, Amec Foster Wheeler, Kings Point House, Queen Mother Square, Poundbury, Dorchester, Dorset \\ DT1 3BW, UK
}

\begin{abstract}
The ANSWERS Software Service is developing a number of techniques to better understand and quantify uncertainty on calculations of the neutron multiplication factor, k-effective, in nuclear fuel and other systems containing fissile material. The uncertainty on the calculated k-effective arises from a number of sources, including nuclear data uncertainties, manufacturing tolerances, modelling approximations and, for Monte Carlo simulation, stochastic uncertainty. For determining the uncertainties due to nuclear data, a set of application libraries have been generated for use with the MONK10 Monte Carlo and the WIMS10 deterministic criticality and reactor physics codes. This paper overviews the generation of these nuclear data libraries by Latin hypercube sampling of JEFF-3.1.2 evaluated data based upon a library of covariance data taken from JEFF, ENDF/B, JENDL and TENDL evaluations. Criticality calculations have been performed with MONK10 and WIMS10 using these sampled libraries for a number of benchmark models of fissile systems. Results are presented which show the uncertainty on k-effective for these systems arising from the uncertainty on the input nuclear data.
\end{abstract}

\section{Introduction}

Amec Foster Wheeler incorporates the ANSWERS Software Service, which supplies high quality software and consultancy services for customers world-wide in the areas of reactor physics, radiation shielding, dosimetry, nuclear criticality and nuclear data.

The ANSWERS Monte Carlo code MONK [1] is used for nuclear criticality safety and reactor physics analysis. It can be run using point energy or multi-group nuclear data libraries. To run in point energy mode, MONK uses the BINGO collision processor and an associated point energy BINGO nuclear data library.

The ANSWERS deterministic code WIMS [2] is used in core physics calculations for numerous reactor types. It uses multi-group nuclear data libraries, usually in the XMAS-172 group structure. These WIMS libraries are also used by MONK in multi-group calculations.

In addition, the WIMS library contains so-called 'burnup' data for use by WIMS and MONK. These include decay constants for short-lived nuclides, fission product yields and capture branching ratios.

The BINGO and WIMS libraries contain equivalent data for around 300 nuclides, allowing a wide range of nuclear systems to be modelled.

ANSWERS has developed methods and data for use in the quantification of uncertainties in the results obtained from the ANSWERS codes. A major source of uncertainty comes from the nuclear data including neutron reaction cross-sections, fission neutron energy and multiplicity and the decay of radionuclides.
In order to quantify the uncertainty arising from the nuclear data for criticality calculations, sets of BINGO and WIMS nuclear data libraries have been generated by sampling JEFF-3.1.2 data using available covariance data and uncorrelated uncertainties (where covariance data is not available). The uncertainty is quantified from the spread in results from MONK and/or WIMS calculations performed with the sampled libraries, for the specific nuclear system of interest.

\section{Standard library production}

\subsection{BINGO library}

BINGO libraries contain continuous energy nuclear data with cross-sections represented as a smoothly varying function of energy and tabulated on an energy grid that allows linear interpolation with a maximum interstitial error in the cross-section of $0.1 \%$.

The evaluated data are processed using the BINGO pre-processor (BPP) code, in order to transform the evaluated data from the ENDF-6 format to a form that allows efficient Monte Carlo sampling. Point crosssections are generated using an external processing 'engine', usually the NJOY99.364 [3] RECONR and BROADR modules. The outgoing angle and energy data for neutrons are transformed into equi-probable bins or probability functions. The $s(\alpha, \beta)$ thermal scattering data are transformed to ease bivariate sampling in momentum and energy, allowing MONK to generate secondary energy data for emitted neutrons during the run. In the resolved resonance range, the point energy representation is adequate to model resonance shielding effects. In the unresolved resonance range, a small number of nuclides,

\footnotetext{
a e-mail: tim.ware@amecfw.com
}

(C) The Authors, published by EDP Sciences. This is an Open Access article distributed under the terms of the Creative Commons Attribution License 4.0 (http://creativecommons.org/licenses/by/4.0/). 
including Th-232, U-233, U-238 and $\mathrm{Pu}-239$, require processing with the sub-group method [1]. Here, group data are generated at infinite dilution and at an appropriate level of resonance self-shielding. Pairs of sub-groups are formed and adjusted to preserve the infinite dilute and shielded cross-sections. This effectively moves the resonance peaks to one of the sub-groups forcing a flux depression dependent on the level of shielding, avoiding the need to sample cross-sections in the unresolved resonance region.

\subsection{WIMS library}

WIMS libraries contain grouped nuclear data following the XMAS-172 group structure. The group averaged crosssections and secondary energy data are generated with the NJOY sequence of modules MODER, RECONR, BROADR, THERMR, PURR and GROUPR. The data are transformed to the current 'WIMS9' library structure using the WIMSR module and a bespoke post-processing code. A resonance shielding treatment is implemented for 38 nuclides including isotopes of $\mathrm{Cu}, \mathrm{Ag}, \mathrm{Hf}, \mathrm{Hg}, \mathrm{Th}, \mathrm{U}$ and $\mathrm{Pu}$. For these nuclides, fine 2400-group cross-sections are generated for the 24 "resonance" energy groups and shielded absolute cross-sections generated for a number of background cross-section values (which are interpolated at runtime).

\section{Covariance library}

A covariance data library has been generated for use with MONK and WIMS. The covariance library can be used in the quantification of nuclear data uncertainties by application in conjunction with the sensitivity methods available in these codes as well as in the generation of sampled nuclear data libraries. The NJOY modules ERRORJ and COVR were used to process the evaluated covariance data into BOXER format in 44 energy groups. The group scheme is a revised version of the SCALE 6.1 44-group scheme, with some group boundaries adjusted to be coincident with the standard WIMS 172-group bounds.

The covariance library contains what was judged the best available data for each nuclide (as of September 2015). The data were taken from JEFF-3.2, ENDF/BVII.1, JENDL-4.0 and TENDL-2011. A small number of nuclides used data from JEFF-3.1.2, JENDL-3.3 or CENDL-3.1. Data taken from JEFF-3.2 include H-1, B-10, C-nat and Fe-56. Data taken from ENDF/B-VII.1 include Zr, U-235, U-238 and Pu-239.

\section{Sampled library production}

\subsection{Sampling method}

All uncertain parameters have been assumed independent and to follow a normal distribution, of known mean and standard deviation. Correlations between variables are then recovered, where covariance data exist for a nuclide, by transforming the uncorrelated random numbers using the Cholesky

decomposition of the covariance matrix. The Cholesky matrix is the lower triangular matrix, $\mathrm{L}$ of the positivedefinite covariance matrix $\mathrm{V}$, such that $\mathrm{V}=\mathrm{L} . \mathrm{L}^{\mathrm{T}}$.

Two sampling methods were considered for producing sets of random numbers. Monte Carlo (random) sampling was the simplest to implement; a random number generator and transformation is used to obtain random variables from the required distribution. One advantage of the Monte Carlo sampling is that of the complete set of libraries generated, the user can use any subset of these libraries as representative of a smaller sample for testing purposes. Increasing the size of the subset should lead to a convergence in the estimate of uncertainty on keffective associated with nuclear data. A disadvantage of the Monte Carlo sampling is potentially many hundreds of libraries could be required to adequately cover the sample space and therefore give the best estimate of the uncertainty due to the nuclear data.

An alternative sampling method, used here, is Latin hypercube sampling (LHS) [4]. Prior to sampling, the cumulative distribution for each variable is divided into equi-probable intervals (the number of intervals must be the same for all variables). Random sampling is then performed, however once an interval has been sampled, it cannot be sampled again. This results in the set of sample points being more representative of the real variability in the uncertain parameters than Monte Carlo sampling. Rather than requiring hundreds of libraries, LHS can cover $95 \%$ of the sample space with $95 \%$ probability with 59 libraries. $97 \%$ probably of $97 \%$ coverage can be achieved with 116 libraries (cf. Wilks' formula [5]).

A disadvantage of the LHS method is that a subset of LHS nuclear data libraries cannot be a drawn from a larger set of LHS libraries; for each number of samples, a new set of libraries must be created.

\subsection{Application of sampling}

Latin hypercube sampling was performed on nuclear data for all nuclides on the BINGO and WIMS libraries. For each energy group of each reaction of each nuclide, a scaling factor is generated by adding the correlated random number, defined above, to unity. The seeds for the random number generation were taken from the system clock at runtime and stored in order that the random numbers could be regenerated in future if required.

Where covariance data exist for a nuclide, the number of variables in the LHS was the sum of covariance energy groups over all reaction types (typically 44 groups for high-fidelity covariance data and fewer groups for threshold reactions and where the covariance terms are constant across two or more energy groups).

For nuclides with limited covariance data, where a reaction has no data for some (or all) energy groups, the LHS used a normal distribution with a standard deviation of $10 \%$. For nuclides with no covariance data, this standard deviation was $25 \%$ for all 44 energy groups.

In order to avoid 'excessive' perturbation resulting in negative macroscopic cross-sections for the overall system, limits were placed on the scaling factors of 0.1 and 10.0 . This avoids errors processing the cross-sections in the applications code but can introduce a marginal negative bias on the average calculated k-effective, as truncating the scaling of small cross-sections with large uncertainties can slightly increase neutron absorption.

\subsection{Sampled library production}

To produce sampled cross-section data on the BINGO and WIMS libraries, the NJOY RECONR module was used 
to generate a PENDF file containing the crosssections reconstructed from the evaluated file at $0{ }^{\circ} \mathrm{K}$. In this way, the same PENDF file can be reintroduced to both the BINGO and WIMS library routes, starting with the NJOY BROADR module.

Modifications were made to the BPP and the WIMS route to accept a pre-generated PENDF. In the WIMS route, the UNRESR module was used rather than PURR for the unresolved resonance treatment, in order to reduce run time.

For each nuclide, the pre-generated PENDF file was perturbed by applying the set of scaling factors to the point-wise cross-sections to produce a set of sampled PENDF files.

In addition to cross-section data, the fission neutron multiplicity and energy spectra data for actinides are sampled in a similar manner. Here, the ENDF files are sampled directly to produce a set of sampled ENDF files, which each have a corresponding sampled PENDF file. The library generation route (as described above) is then resumed using the sampled PENDF (and for actinides, ENDF) files.

In order to estimate the uncertainty on the thermal scattering cross-section of bound nuclides (e.g., $\mathrm{H}$ in $\mathrm{H}_{2} \mathrm{O}$, $\mathrm{H}$ in $\mathrm{CH}_{2}, \mathrm{C}$ in graphite), the uncertainties on the elastic scattering cross-sections were used as a best-available estimate, as covariances on bound thermal scattering are not yet available. In BINGO and WIMS, the bound thermal scattering is used in place of the elastic scattering below $4 \mathrm{eV}$. Due to low fidelity of the covariance data at thermal energies for bound scatterers, the scaling factors below $4 \mathrm{eV}$ are constant (and temperature-independent). Therefore, a single scaling factor can be used for each sample of each nuclide. For the BINGO library, the thermal scattering data are formatted as point-wise cross-sections and outgoing energy distributions. Therefore, the scaling factors could be applied after the cross-sections are formed by automated editing of the final data file. For WIMS, the NJOY THERMR module was extended to apply a scaling factor to the reconstructed thermal scattering crosssections prior to output.

Perturbed decay constants are added to the WIMS library via sampling of the evaluated half-life and its uncertainty. Fission product yields are sampled using only the available uncertainties, noting that the current lack covariances is believed to have a significant effect). Capture branching ratios are sampled using the uncertainties available on the thermal capture crosssections for the required nuclides.

\subsection{Libraries produced}

Complete sets of data libraries were produced in the BINGO and WIMS formats. Latin hypercube sampling of JEFF3.1.2 evaluated data was performed with the sample space divided into 25 intervals (LHS25) and 60 intervals (LHS60).

BINGO library data were generated at a standard set of temperatures: 293.6, 500, 1000, 1500, 2000 and $3500 \mathrm{~K}$. Data for intermediate temperatures are calculated by MONK at run time using an accelerated Doppler broadening method. The WIMS libraries were at a standard set of temperatures: 293.16, 573.16, 973.16,

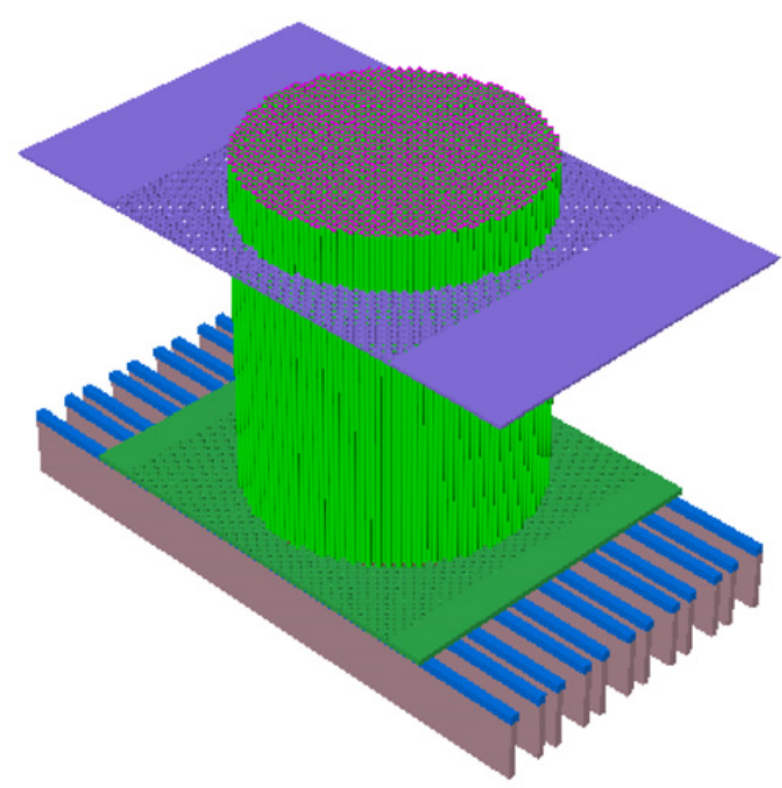

Figure 1. Visual Workshop image of DIMPLE model.

1473.16 and $2973.16 \mathrm{~K}$, with intermediate temperatures obtained by interpolation.

\section{Library testing}

The sampled BINGO and WIMS libraries were tested with the MONK10 and WIMS10 codes. In addition to basic function testing at all library temperatures with a simple $\mathrm{UO}_{2}$ pincell model, calculations of k-effective for criticality benchmark experiments were performed. These cases were taken from the MONK and WIMS code validation databases.

The DIMPLE assembly SO1 criticality benchmarks, performed at AEE Winfrith (UK), were five configurations (labelled SO1/A to /E) consisting of 1441-1597 pins of $3^{\mathrm{w}} \%$ enriched $\mathrm{UO}_{2}$, moderated by light water, of varying critical height, at room temperature [6]. The MONK models include detailed 3D geometry, as illustrated in Fig. 1. The WIMS models include the array of fuel pins with appropriate leakage modelled.

The BICEP criticality benchmarks, performed at AERE Harwell (UK), were $0.42-1.14{ }^{\text {w }} \%$ depleted, natural and enriched U-metal pins, air-cooled in graphite "stacks" at room temperature [7]. The fuel enrichment, pin diameter and lattice pitch varies between experiments. Both MONK and WIMS model these exponential experiments as a single fuel pin within an air gap and graphite cuboid, with reflection on all external boundaries. Leakage is applied to the infinite pincell model using experimentally derived buckling terms.

MONK10 calculations were performed with the BINGO libraries and WIMS libraries (labelled in the results as 'NOVICE'). The stochastic uncertainty on $\mathrm{k}$-effective for individual calculations is $100 \mathrm{pcm}$ $(\sigma=0.001)$.

Figure 2 illustrates the spread in the calculated k-effective resulting from use of the set of sampled data libraries. For each individual library, the results from MONK and WIMS are in good agreement (within $2 \sigma$ ) which is as expected due to the use of the same underlying 


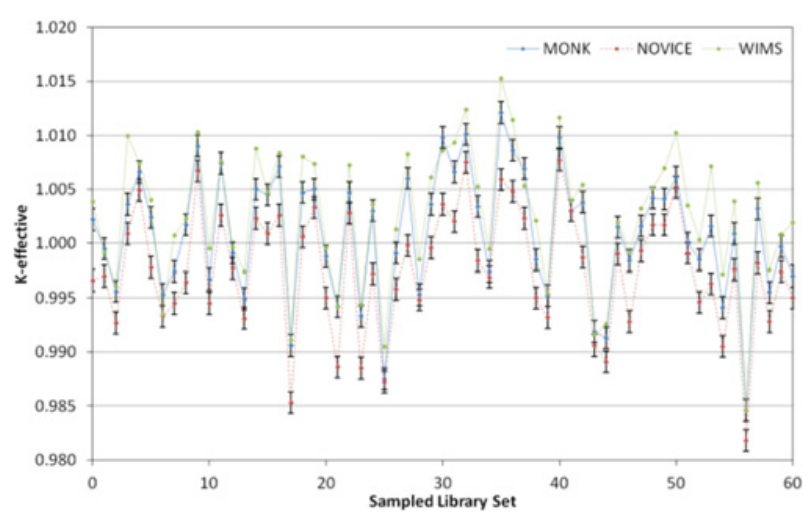

Figure 2. Calculated k-effective for DIMPLE SO1/A assembly.

Table 1. Std. dev. in k-effective (averaged by benchmark set).

\begin{tabular}{|c|c|c|c|}
\hline Code & Sampling & DIMPLE cases & BICEP cases \\
\hline MONK & LHS25 & $550 \mathrm{pcm}$ & $720 \mathrm{pcm}$ \\
\hline MONK & LHS60 & $580 \mathrm{pcm}$ & $760 \mathrm{pcm}$ \\
\hline NOVICE & LHS25 & $560 \mathrm{pcm}$ & $720 \mathrm{pcm}$ \\
\hline NOVICE & LHS60 & $560 \mathrm{pcm}$ & $760 \mathrm{pcm}$ \\
\hline WIMS & LHS25 & $600 \mathrm{pcm}$ & $710 \mathrm{pcm}$ \\
\hline WIMS & LHS60 & $610 \mathrm{pcm}$ & $760 \mathrm{pcm}$ \\
\hline
\end{tabular}

sampled nuclear data. Sampled library set '0' represents the unsampled data.

The uncertainty on k-effective due to the sampling of the nuclear data is summarised in 6 . Here the standard deviation in the results from the five DIMPLE cases and the 12 BICEP cases have been averaged; the standard deviations for individual cases are broadly consistent.
These results demonstrate that the LHS nuclear data libraries can estimate the nuclear data uncertainty on the k-effective for different nuclear systems. Some specific applications may take the uncertainty arising from the nuclear data as three times the standard deviation in the calculation results.

\section{Conclusion}

The LHS library method for uncertainty quantification is independent of the specific systems to be analysed and does not require changes to the physics codes. It gives reasonable estimates of the nuclear data uncertainty on k-effective and is readily applied to the uncertainty analysis of other quantities such as reactor power distributions or radiation shielding effectiveness.

\section{References}

[1] ANSWERS Software Service, ANSWERS/MONK/ REPORT/009 (2014)

[2] ANSWERS Software Service, ANSWERS/WIMS/ REPORT/014 (2014)

[3] R.E. MacFarlane, D.W. Muir, LA-12740-M (1994)

[4] M.D.McKay, R.J.Beckman, W.J.Conover, Technometrics 21, 239-245 (1979)

[5] S.S. Wilks, Ann. Math. Statist. 12, 91-96 (1941)

[6] D. Hanlon, LEU-COMP-THERM-048, ICSEP Handbook NEA/NSC/DOC(95)03/IV (2002)

[7] British Industries Collaborative Exponential Programme, AEEW-R235 (1963) 